\title{
Influence of Spinal Cord Integrity on Gait Control in Human Spinal Cord Injury
}

\author{
Neurorehabilitation and \\ Neural Repair \\ 2016, Vol. 30(6) 562-572 \\ (C) The Author(s) 2015 \\ Reprints and permissions: \\ sagepub.com/journalsPermissions.nav \\ DOI: I0.1 I77/I5459683I5600524 \\ nnr.sagepub.com
}

(SAGE

\author{
Lea Awai, PhD', Marc Bolliger, PhD', Adam R. Ferguson, PhD', \\ Grégoire Courtine, PhD $^{3}$, and Armin Curt, $\mathbf{M D}^{\prime}$
}

\begin{abstract}
Background. Clinical trials in spinal cord injury $(\mathrm{SCl})$ primarily rely on simplified outcome metrics (ie, speed, distance) to obtain a global surrogate for the complex alterations of gait control. However, these assessments lack sufficient sensitivity to identify specific patterns of underlying impairment and to target more specific treatment interventions. Objective. To disentangle the differential control of gait patterns following $\mathrm{SCl}$ beyond measures of time and distance. Methods. The gait of 22 individuals with motor-incomplete $\mathrm{SCl}$ and 21 healthy controls was assessed using a high-resolution 3-dimensional motion tracking system and complemented by clinical and electrophysiological evaluations applying unbiased multivariate analysis. Results. Motor-incomplete SCl patients showed varying degrees of spinal cord integrity (spinal conductivity) with severe limitations in walking speed and altered gait patterns. Principal component (PC) analysis applied on all the collected data uncovered robust coherence between parameters related to walking speed, distortion of intralimb coordination, and spinal cord integrity, explaining $45 \%$ of outcome variance (PC I). Distinct from the first PC, the modulation of gaitcycle variables (step length, gait-cycle phases, cadence; PC 2) remained normal with respect to regained walking speed, whereas hip and knee ranges of motion were distinctly altered with respect to walking speed (PC 3). Conclusions. In motorincomplete $\mathrm{SCl}$, distinct clusters of discretely controlled gait parameters can be discerned that refine the evaluation of gait impairment beyond outcomes of walking speed and distance. These findings are specifically different from that in other neurological disorders (stroke, Parkinson) and are more discrete at targeting and disentangling the complex effects of interventions to improve walking outcome following motor-incomplete $\mathrm{SCl}$.
\end{abstract}

\section{Keywords}

spinal cord injury, human, gait, motor control

\section{Introduction}

More than half of patients with spinal cord injury (SCI) exhibit incomplete injuries, leading to varying degrees of functional impairment of the affected limbs. ${ }^{1}$ The provision of patient-customized interventions ${ }^{2}$ for motor-incomplete spinal cord injury (iSCI) is limited by the rather crude clinical tools currently used for assessing locomotion (eg, motor scores, speed, distance). Detailed analysis of locomotion in iSCI patients serves 2 important aims. First, the ability to score locomotor function allows the determination of changes in walking capacity to measure the effect of therapeutic interventions. $^{3-6}$ Second, comprehensive assessment of walking beyond unrefined measures of capacity may provide insights into mechanisms underlying changes of neural control.,

The majority of studies on gait recovery after iSCI focus on quantitative measures of time and distance $(10-\mathrm{m}$ walk test, 6-minute walk test), functional scores (eg, Walking Index for Spinal Cord Injury, and the mobility subscore of the Spinal Cord Independence Measure $)^{9-11}$ or a subjective rating of gait quality. ${ }^{12}$ These investigations mainly assessed the temporal profiles of ambulatory capacity ${ }^{13}$ and the impact of rehabilitation interventions. ${ }^{4,14}$ Few studies have used more detailed kinematic methodologies (eg, quality of walking) to uncover complex alterations of iSCI walking. ${ }^{15-18}$ The objective of the present study was to establish a comprehensive assessment framework in iSCI, applying multivariate data analysis of motor function to (1) reveal the influence of spinal cord integrity on walking outcomes and (2) provide a new approach for disentangling different domains of locomotor control in human SCI.

'Spinal Cord Injury Center, Balgrist University Hospital, Zurich, Switzerland

${ }^{2}$ Brain and Spinal Injury Center (BASIC), University of California - San Francisco (UCSF), CA, USA

${ }^{3}$ Center for Neuroprosthetics and Brain Mind Institute, Swiss Federal Institute of Technology (EPFL), Lausanne, Switzerland

Corresponding Author:

Lea Awai, PhD, Spinal Cord Injury Center, Balgrist University Hospital, Forchstrasse 340, CH-8008 Zürich, Switzerland.

Email: lawai@paralab.balgrist.ch 
Table I. Demographic and Clinical Characteristics of Patients.

\begin{tabular}{|c|c|c|c|c|c|c|}
\hline ID & Age, years & Sex & Cause of Injury & Level of $\mathrm{SCl}$ & LEMS & Assistive Device \\
\hline 01 & 73 & $M$ & Traumatic & $\mathrm{C5}$ & 24.5 & Wheeler \\
\hline 02 & 24 & $M$ & Traumatic & $\mathrm{C} 3$ & 24.5 & - \\
\hline 03 & 36 & $\mathrm{~F}$ & Disc prolapse & $\mathrm{T} 7 / 8$ & 16.0 & Crutches \\
\hline 04 & 47 & $\mathrm{~F}$ & Spinal ischemia & $\mathrm{T} 9 / 10$ & 24.0 & - \\
\hline 05 & 30 & $M$ & Traumatic & L2 & 22.5 & - \\
\hline 08 & 60 & $\mathrm{~F}$ & Spinal ischemia & T5 & 23.0 & Wheeler \\
\hline 09 & 48 & $\mathrm{~F}$ & Traumatic & T7 & 15.0 & One cane \\
\hline 10 & 39 & $\mathrm{~F}$ & Spinal myelitis & $C, T$ & 19.0 & Wheeler \\
\hline 12 & 65 & $M$ & Hematoma & $\mathrm{C} 6$ & 24.0 & - \\
\hline 13 & 23 & $M$ & Traumatic & $\mathrm{C7}$ & 9.5 & Wheeler \\
\hline 15 & 78 & $M$ & Diverse & $\mathrm{C} 3 / 4$ & 22.5 & Wheeler \\
\hline 16 & 60 & $M$ & Spinal canal stenosis & $\mathrm{T} 9 / 10$ & 25.0 & - \\
\hline 17 & 55 & $M$ & Cervical myelopathy & C5 & 19.5 & - \\
\hline 18 & 63 & $\mathrm{~F}$ & Epidural abscess & $\mathrm{T} 4$ & 23.0 & Crutches \\
\hline 19 & 43 & $M$ & Traumatic & $\mathrm{C} 2$ & 24.0 & Two canes \\
\hline 20 & 61 & $M$ & Disc prolapse & $\mathrm{C} 2$ & 25.0 & - \\
\hline 21 & 40 & $M$ & Traumatic & $\mathrm{C5}$ & 22.5 & - \\
\hline 22 & 64 & $M$ & Spondylitis, abscess & $\mathrm{T} 4$ & 23.5 & - \\
\hline 23 & 32 & $M$ & Traumatic & TII & 8.0 & Crutches \\
\hline 24 & 36 & M & Traumatic & $L 4 / 5$ & 23.5 & - \\
\hline 25 & 41 & $M$ & Traumatic & $\mathrm{C7}$ & 24.0 & - \\
\hline 26 & 48 & $\mathrm{~F}$ & Disc prolapse & TIO & 24.0 & Two sticks \\
\hline
\end{tabular}

Abbreviations: C, cervical; F, female; ID, patient identification number; L, lumbar; LEMS, lower-extremity motor score (mean value of left and right; maximum $=25$ ); $\mathrm{M}$, male; $\mathrm{SCl}$, spinal cord injury; $\mathrm{T}$, thoracic.

\section{Materials and Methods}

\section{Participants}

Inclusion criteria: iSCI patients aged 18 years and older, who were at least able to stand and walk without the assistance of another person, were included.

Exclusion criteria: Patients suffering from any other neurological disorder or preexisting gait impairment were excluded. The study was approved by the Zurich Cantonal ethics committee in accordance with the Declaration of Helsinki. Participants provided written informed consent.

The data of 22 iSCI patients ( 15 men, 7 women; $48.3 \pm$ 15.6 years old; weight, $74.6 \pm 13.4 \mathrm{~kg}$; height, $171.6 \pm 8.4$ $\mathrm{cm}$ ) and 21 healthy controls ( 8 men, 13 women; $38.4 \pm 14.3$ years old; weight, $66.7 \pm 12.1 \mathrm{~kg}$; height, $172.2 \pm 8.2 \mathrm{~cm})$ were included in this study (Table 1).

\section{Experimental Setup}

Participants walked barefoot overground, along a straight 8-m walkway and on a treadmill at different walking speeds. Patients were allowed to use an assistive device for overground walking if needed and were allowed to hold handrails on the treadmill. Participants first walked overground to assess their preferred walking speed. This value was used to subsequently determine the relative walking speeds on the treadmill: $50 \%$ (slow), $100 \%$ (preferred), and $150 \%$ (fast) of preferred speed. Additionally, the participants walked at predefined treadmill speeds, starting at a slow speed $(0.5 \mathrm{~km} / \mathrm{h})$, and increased speeds in steps of $0.5 \mathrm{~km} / \mathrm{h}$ until $150 \%$ of preferred speed (fast) was reached. If a patient could not reach this speed, the maximal walking speed they were able to walk was recorded instead.

For lower-body kinematics, 8 infrared cameras (T10, Vicon motion systems Ltd, Oxford, UK) at $200 \mathrm{~Hz}$ and 2 synchronized digital high-speed video cameras (pilot series, Basler AG, Ahrensburg, Germany) at $100 \mathrm{~Hz}$ were used. Pressure sensors underneath the treadmill belt (Zebris FDM-T, zebris Medical GmbH, Isny im Allgäu, Germany) recorded force distributions at $120 \mathrm{~Hz}$. Also, 16 reflective markers (16 $\mathrm{mm}$ in diameter) were placed on bony landmarks, according to the Vicon Plug-in Gait lower-body model.

\section{Outcome Measures}

Time-Distance Parameters. The following time-distance parameters were determined: preferred overground walking speed (preferred speed, $\mathrm{km} / \mathrm{h}$ ), step length $(\mathrm{cm})$, cadence (strides/min), stance phase (\%), and single support phase 
(\%) during treadmill walking. Initial foot contact defined gait-cycle onset. One gait cycle ranged from initial contact of one foot to the subsequent initial contact of the same foot. Time-distance parameters were calculated by the Zebris WinFDM-T software.

Kinematic Data. Kinematic data were first processed offline using the Vicon Nexus Software (1.5.2) to fill data gaps and apply the Vicon Plug-in Gait kinematic model. Data were exported for further analysis using custom-written MATLAB scripts (MATLAB R2010b/R2013a, Mathworks Inc, Natick, MA): continuous data from $\sim 20$ consecutive gait cycles were cut into individual gait cycles and time-normalized using linear interpolation. The maximal range of motion (ROM) of the hip and knee angles, averaged over all gait cycles, and the resultant velocity vector (angular change per unit time) of these 2 angles at toe-off (angular velocity at toe-off) were calculated. To assess whether angular velocity at toe-off limits walking speed in patients, their data were compared with those of controls at patients' maximal speed by interpolating data of controls using a thirddegree polynomial least-squares fit. Variable $\Delta$ values reflect the difference between slow and preferred speed, providing a measure for the modulatory capacity of different features of gait with a doubling of walking speed (ie, from $50 \%$ to $100 \%$ of preferred speed). To evaluate multisegmental intralimb coordination, combined hip-knee angle-angle plots, called cyclograms, were studied. The angular component of coefficient of correspondence $(\mathrm{ACC})^{19}$ was calculated to quantify the cycle-to-cycle cyclogram shape consistency. To quantify cyclogram quality, the shape difference between patients' cyclograms and a norm cyclogram of healthy individuals at preferred speed was calculated using the square root of sum of squared distances $\left(\mathrm{SSD}^{20}\right)$ :

$$
\operatorname{SSD}_{j, k}=\sqrt{\sum\left[\left(\alpha_{j, i}-\alpha_{k, i}\right)^{2}+\left(\beta_{j, i}-\beta_{k, i}\right)^{2}\right]},
$$

where cyclogram $j$ (patient) is compared with cyclogram $k$ (norm cyclogram) after uniform scaling and translation of the centroid to the origin, and $\alpha$ and $\beta$ represent hip and knee angles, respectively.

Clinical and Electrophysiological Data. Lower-limb voluntary muscle strength was measured by trained examiners using the American Spinal Injury Association (ASIA) lowerextremity motor score. The mean of both legs was evaluated (maximum $=25$ ). Spinal cord integrity was assessed via electrophysiological recordings according to clinical routine by motor-evoked potentials (MEPs) of the tibialis anterior and abductor hallucis muscles, and somatosensoryevoked potentials (SSEPs) were elicited from the tibialis posterior nerve. MEPs were elicited by single-pulse transcranial magnetic stimulation of respective contralateral motor cortex areas just next to the midline via a figure-ofeight coil connected to a magnetic stimulator (The Magstim Company Ltd, Wales, UK). SSEPs were elicited dorsal of the malleolus medialis, and the recording electrodes were placed in the $\mathrm{Cz}^{\prime}-\mathrm{Fz}$ configuration according to the international 10/20 system. P40/N50 peaks were chosen for SSEP evaluation. SSEPs and MEPs were evaluated by taking into account both response latency and amplitude, as reported previously for SSEPs, ${ }^{21}$ resulting in a 5-point impairment scale.

\section{Statistical Analysis}

Statistical analyses were performed using SPSS Statistics 19 (IBM Corp, New York, NY) and MATLAB.

Principal Component Analysis (PCA). To account for the multidimensionality of data, a categorical PCA was performed, which included 23 parameters of all control and iSCI participants, to discern clusters of interrelated parameters of walking, leveraging the extensive ensemble of gait-related variables. ${ }^{22}$ Principal components (PCs) were retained according to the Scree plot $^{23}$ and PC overdetermination (at least 2 |factor loadings $\mid \geq 0.6$ ). Variables with high loadings on the same PC indicate a strong interrelationship. In the context of kinematic outcomes, PCs have been interpreted to reflect neurobiological "motor primitives" or discretely organized "motor modules" of complex movements. ${ }^{24}$ To examine the validity and robustness of the PC clusters, a bootstrapping procedure was used with 10000 iterations on a random subset of $80 \%$ of participants, and the factor loadings were crossvalidated using consensus from Pearson's $r$, root mean square (RMS), coefficient of congruence (CC), and Cattell's S. Monte Carlo studies have indicated that consensus of R, CC, RMS, and $\mathrm{S}$ together indicate robust converging support for stability of multidimensional patterns, independent of the number of subjects used. Here, we use these statistics in our bootstrapping algorithms to reveal that the PC loading patterns are highly robust, despite the limited number of subjects available for SCI clinical populations. ${ }^{25,26}$

Pairwise Comparisons. Spearman's rank correlation coefficient $\rho$ was used to correlate a pair of single outcome measures. The comparison of 2 groups was performed by a 2-tailed $t$ test for normally distributed data and a MannWhitney $U$ test or Wilcoxon signed-rank test for nonnormally distributed independent or dependent samples, as appropriate. Bonferroni correction was applied $(0.05 / \mathrm{n})$.

\section{Results}

The gait pattern of healthy controls and iSCI patients was studied both qualitatively and quantitatively considering specific characteristics of lower-limb coordination. Gait 

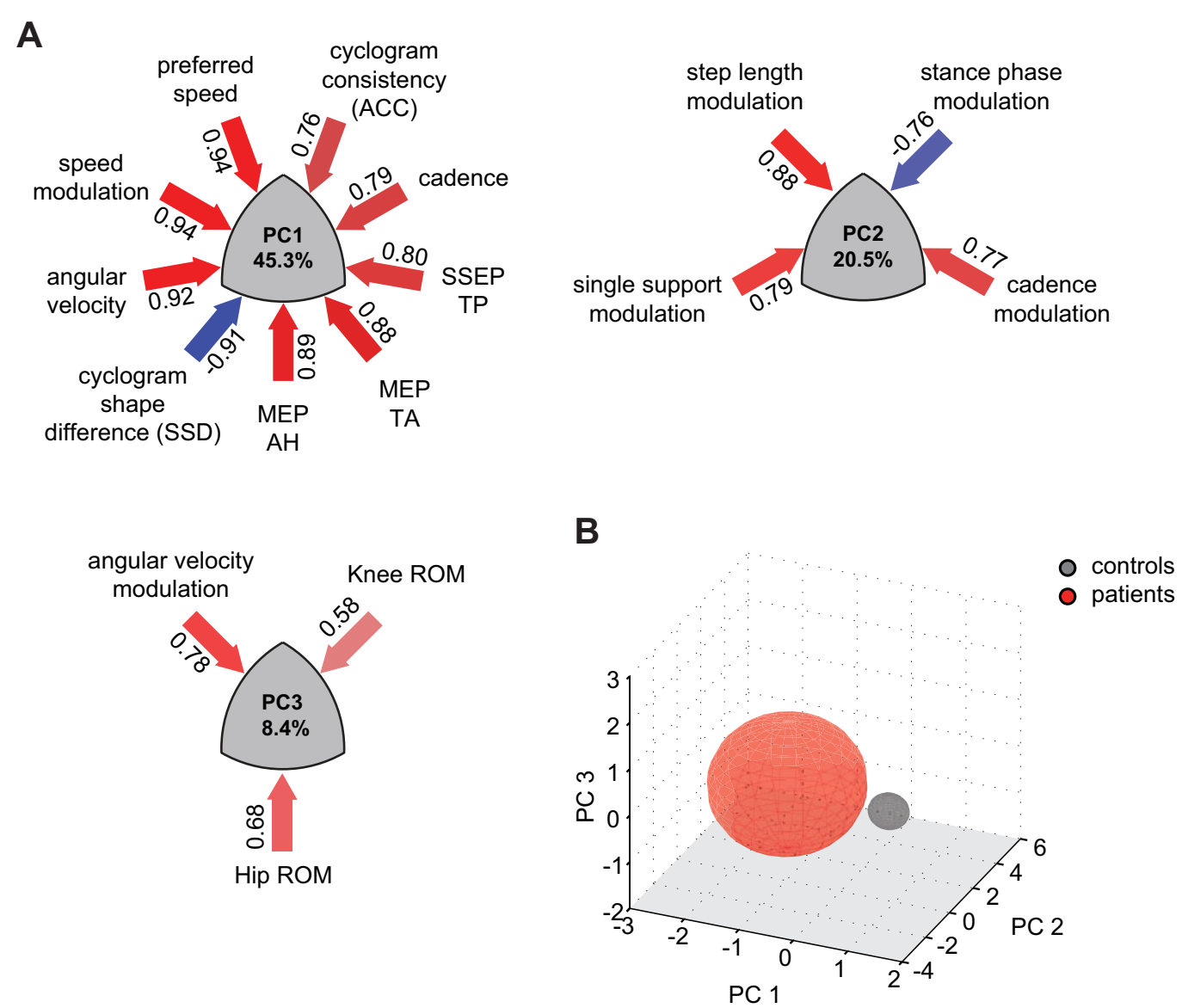

Figure I. Multivariate analysis of gait-related parameters: principal components (PCs) I to 3 explain $74.2 \%$ of the total variance. PCs show clustered variance of multiple parameters with high loadings on the corresponding PC. A. Variable loadings are depicted in numbers next to the arrows, whose colors reflect the magnitude and relationship of loading (positive relationship $=$ red, negative $=$ blue). Transformed data are plotted in the 3D space determined by the orthogonal PCs I to 3 and grouped according to neurological condition (B). Abbreviations: ACC, angular component of coefficient of correspondence; AH, abductor hallucis; MEP, motor-evoked potential; ROM, range of motion; SSD, square root of sum of squared distances (cyclogram shape difference); SSEP, somatosensory-evoked potential; TA, tibialis anterior; TP, tibialis posterior.

Note: Color version of the figure is present with the online version of this issue at www.nnr.sagepub.com

pattern features were analyzed at 2 different walking speeds: $0.5 \mathrm{~km} / \mathrm{h}$ and preferred speed. To compare the gait pattern at a matching speed, patients at their preferred speed (mean $=$ $2.0 \mathrm{~km} / \mathrm{h}$ ) were additionally compared with controls walking at $2.0 \mathrm{~km} / \mathrm{h}$.

\section{Multivariate Analysis}

PCA of the 23 variables revealed 3 robustly reproducible PCs $(r \geq 0.98$, RMS $\leq 0.01, \mathrm{CC} \geq 0.97$, Cattell's $\mathrm{S} \geq 0.5)$ that together accounted for $74.2 \%$ of the variance (Figure 1A). The overall variance was mainly influenced by the neurological condition (ie, healthy vs SCI; Figure 1B). PC1 (45.3\% of the variance) was characterized by variables of walking dynamics (eg, walking speed, angular velocity) as well as spinal cord integrity (ie, MEP, SSEP) and quantified metrics of the intralimb coordination (shape difference [SSD], cycle-to-cycle consistency [ACC]). The orthogonal PC2 assembled step length and variables associated with the timing and reciprocal bilateral coordination of the gait cycle. The third PC was predominantly defined by hip and knee ROMs.

\section{Gait Control Impairments}

ANOVA and post hoc Tukey's tests revealed that the 2 groups (iSCI and control group) significantly differed along PC1, but no group distinction was seen with respect to $\mathrm{PC} 2$ or PC3. Measures representing spinal cord integrity (MEP, SSEP) as well as walking speed assembled on PC1 (Figure 1A) and were significantly affected in iSCI participants. Clinically, patients were limited in preferred and 
maximal walking speeds (Figure 2A); whereas all controls were able to increase their speed to $150 \%$ of preferred speed $(6.18 \pm 0.79 \mathrm{~km} / \mathrm{h}), 12$ of 19 patients showed a restricted ability to increase their walking speed beyond 100\% (2.85 \pm $1.55 \mathrm{~km} / \mathrm{h}$ ). Also among the best walkers (preferred speed $\geq 3 \mathrm{~km} / \mathrm{h}$ ), 3 of 5 patients could not attain their relative maximal walking speed. Even more pronounced than speed impediments, patients showed a remarkably limited capacity to modulate angular velocity at toe-off (Figure 2B). At the $0.5 \mathrm{~km} / \mathrm{h}$ speed, the 2 groups showed comparable angular velocities, whereas iSCI participants diverged from control data with increasing walking speed. The increase in angular velocity was significant from $0.5 \mathrm{~km} / \mathrm{h}$ to preferred as well as from preferred to maximal walking speed in control participants (paired $t$ test: $P<.001$ and $P=.003$ ). Patients could only increase the angular velocity between the $0.5 \mathrm{~km} / \mathrm{h}$ and preferred speed but not between preferred and maximal speed (paired $t$ test: $P<.001$ and $P=.355$ ). At patients' maximal speed $(2.85 \mathrm{~km} / \mathrm{h})$, the angular velocity was significantly lower compared with the interpolated values at the respective speed in controls ( $t$ test: $P=.026)$.

The quality of intralimb coordination (shape difference to normal) showed a striking convergence when increasing speed from $0.5 \mathrm{~km} / \mathrm{h}$ to preferred in healthy controls, whereas iSCI patients with a deteriorated pattern were unable to normalize it (Figure 2C). Metrics quantifying hip-knee cyclogram characteristics showed the sensitivity of the intralimb coordination to changes in speed: the cycle-to-cycle consistency (ACC) increased from $0.5 \mathrm{~km} / \mathrm{h}$ to preferred speed in both groups (Wilcoxon signed rank test: $P<.001$ ) but was still higher in controls compared with patients at preferred speed (Mann-Whitney $U$ test: $P<.001$; Figure 2D). However, at $0.5 \mathrm{~km} / \mathrm{h}$ and at the matching speed $(2 \mathrm{~km} / \mathrm{h})$, the ACC was indistinguishable between groups (MannWhitney $U$ test: $P=.394 ; P=.666$ ). The shape difference to normal of the cyclogram (SSD) improved at preferred speed compared with the $0.5 \mathrm{~km} / \mathrm{h}$ walking in both groups (paired $t$ test: $P<.001$ ). However, it remained different from normal in iSCI patients both at a matching speed $(2 \mathrm{~km} / \mathrm{h})$ and when comparing preferred speeds ( $t$ test: $P<.001$; Figure $2 \mathrm{E}$ ), emphasizing the diminished normalization capacity.

\section{Preserved Control of Gait-Cycle Parameters}

In contrast to the variables determining PC1, PC2 was loaded by gait-cycle parameters that remained well modulated according to walking speed (Figures 3A through 3D). The values of step length, cadence, single support, and stance phase were well within the range of controls.

\section{Range of Motion}

Hip ROM and knee ROM represent the maximal sagittal excursion of single joint angles during a gait cycle. At 0.5 $\mathrm{km} / \mathrm{h}$ and at the matching speed $(2 \mathrm{~km} / \mathrm{h})$, iSCI participants showed a greater hip ROM compared with controls, while the knee ROM did not differ between groups ( $t$ test: $P=.005$ and $P=.961$ at $0.5 \mathrm{~km} / \mathrm{h} ; P<.001$ and $P=.613$ at matching speed). In contrast, the hip ROM showed no difference between groups at preferred speed while the knee ROM was reduced in iSCI participants ( $t$ test: $P=.317$ and $P=.001)$.

\section{Speed Dependence}

Some gait variables, for example, angular velocity at toeoff, were linearly related to preferred speed (Figure 4A), where patients attained lower values than controls in accordance with their reduced walking speed. In contrast, the cyclogram shape did not reflect preferred walking speed in healthy participants, given that the SSD remained within a narrow range ( $\sim 2$ to 5$)$ in the control group irrespective of speed (Figure 4B). Patients, on the other hand, showed a high relation between the cyclogram shape and preferred speed. Interestingly, spinal cord integrity (ie, spinal cord conductivity as measured by evoked potentials) did not determine the speed capacity of patients (Figures 4C and 4D). The ROMs showed rather weak relations to preferred speed, especially in iSCI participants but also in healthy individuals (Figures 4E and 4F).

\section{Discussion}

The present study, analyzing combined clinical and kinematic assessments, provides specific findings of the complexity of gait alterations and locomotor control in walking following iSCI. Through statistical data integration across multiple outcome modalities, specific clusters of coherent gait parameters were distinguishable that were related to spinal cord integrity and discretely controlled after iSCI. The findings are specifically different from that for other neurological disorders (eg, stroke and Parkinson). The effectiveness of any treatment intervention (training, drugs) in iSCI to improve walking outcome may be evaluated against these distinguishable clusters of outcome. ${ }^{27}$

\section{Gait Control Impairments}

Clinical (ASIA protocol) and electrophysiological measures (ie, MEPs and SSEPs) have been shown to be sensitive in assessing spinal cord impairment following SCI and are predictive for functional outcome. ${ }^{5,28-30}$ Although these measures are sensitive at predicting levels of walking outcome (no, therapeutic, functional, and full locomotor capacity $^{31,32}$ ), they may not be able to predict more detailed gait characteristics or continuous measures such as walking speed. This is the first study to show that SCI specifically affects certain clusters of parameters while others remain well controlled. Different levels of gait pattern distortion 
A

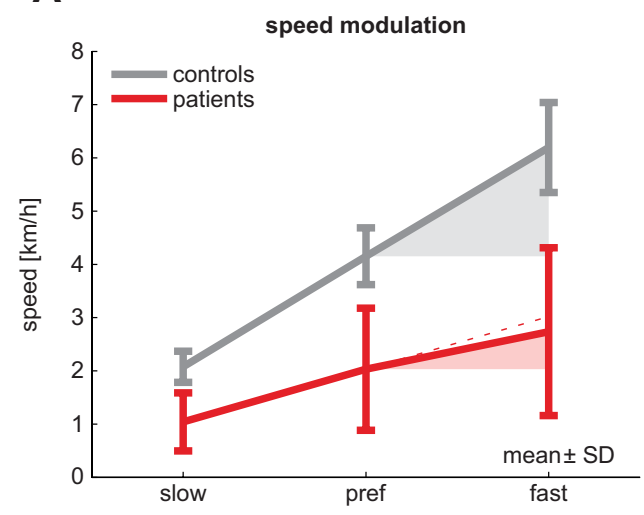

B

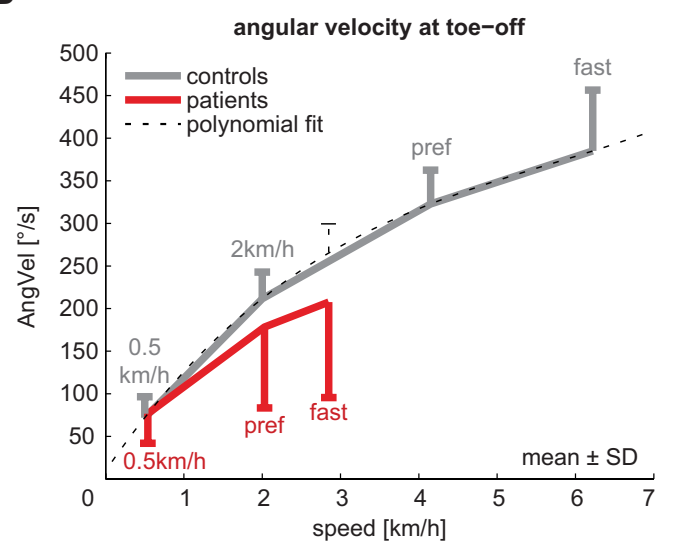

D
C
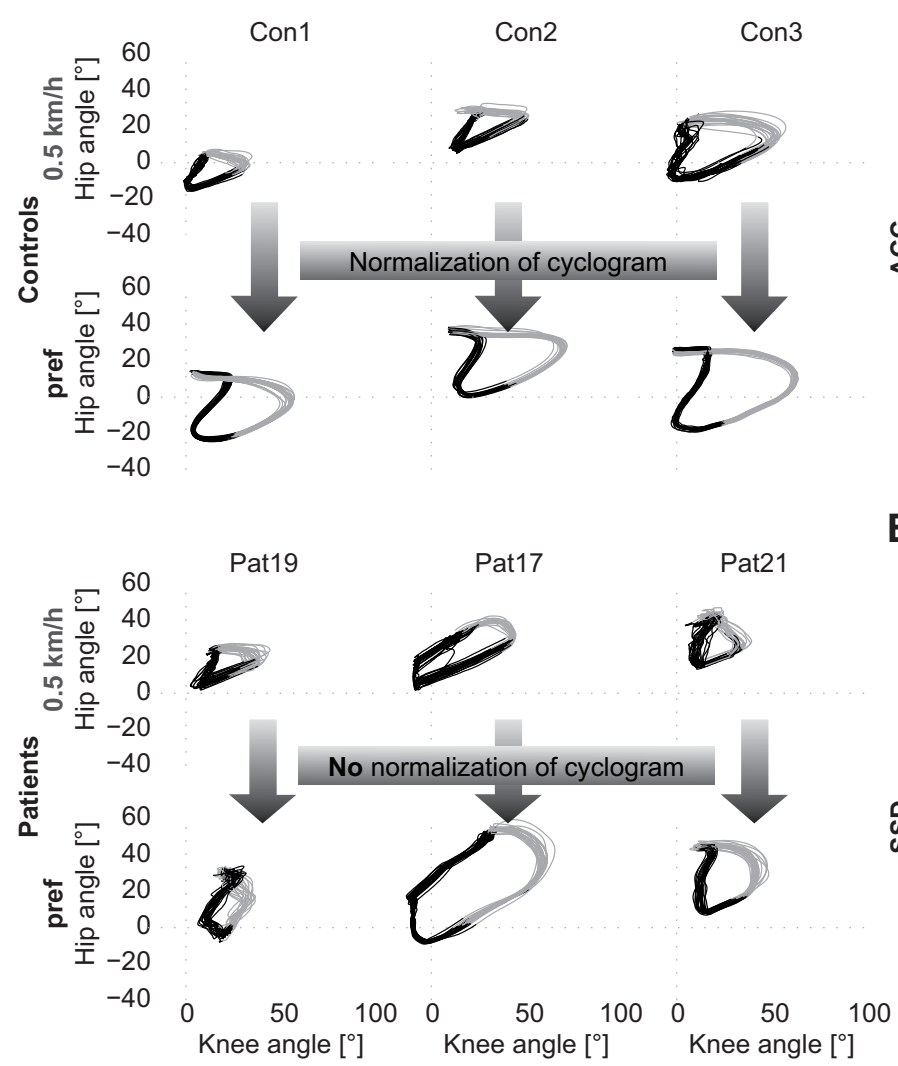

\section{Cyclogram consistency}

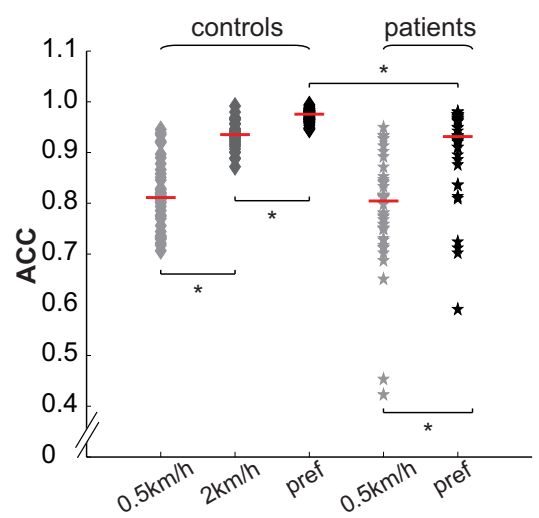

E

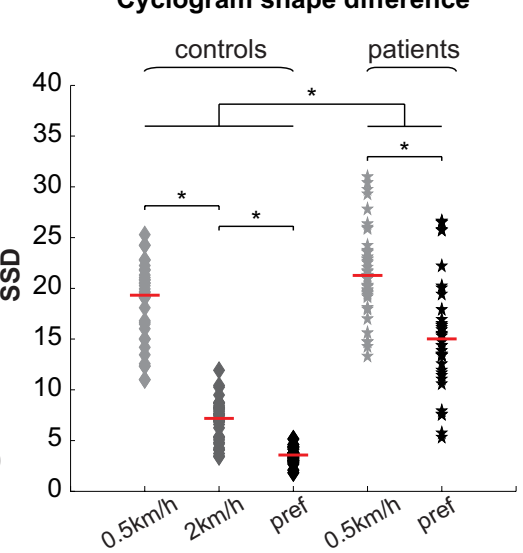

Figure 2. Speed modulation of parameters contributing to $\mathrm{PCl}$ : (A) Absolute speed and speed modulation is limited in iSCl patients. Theoretical linear increase is indicated by the red dotted line. Error bars indicate $\pm I S D$. (B) The diagram illustrates the modulation of the hip-knee angular velocity at toe-off. A third-degree polynomial fit (thin dotted line) was used to interpolate control participants' data to obtain their angular velocity at patients' fast speed. Error bars indicate I SD. (C) Hip-knee cyclograms of controls (top 2 rows) and patients (bottom 2 rows) both at $0.5 \mathrm{~km} / \mathrm{h}$ and at preferred (pref) walking speed are shown; 20 gait cycles are plotted; the black color indicates stance phase, and gray color indicates swing phase. (D) The cycle-to-cycle reproducibility of the hip-knee cyclogram (ACC) improved with increasing speed in both groups but was lower in patients at preferred speed. (E) The shape difference of the cyclogram compared with normal (SSD) improved in patients at preferred speed but remained different from controls at all speeds. Abbreviations: ACC, angular component of coefficient of correspondence; AngVel, angular velocity at toe-off; iSCl, incomplete spinal cord injury; PC, principal component; SD, standard deviation; SSD, square root of sum of squared distances (cyclogram shape difference). $* P<.05$.

Note: Color version of the figure is present with the online version of this issue at www.nnr.sagepub.com 
A

controls
- patients

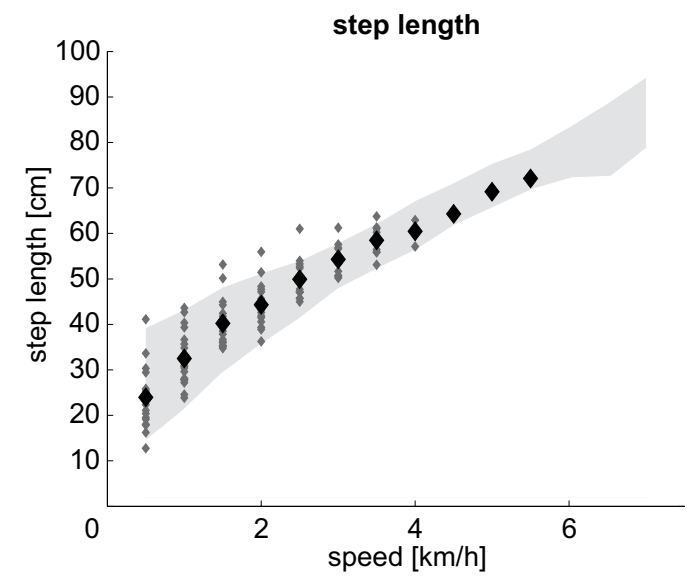

C

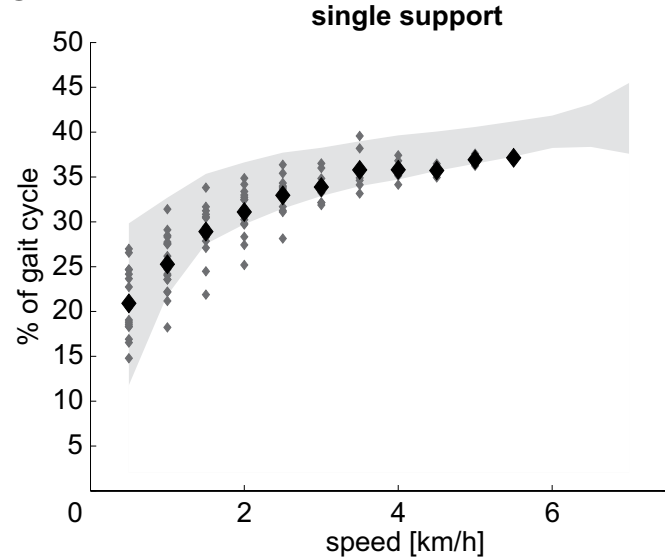

B

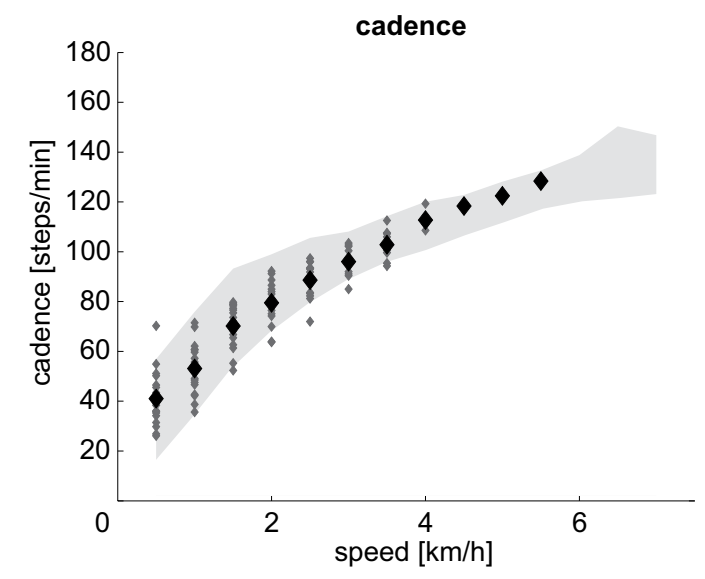

D

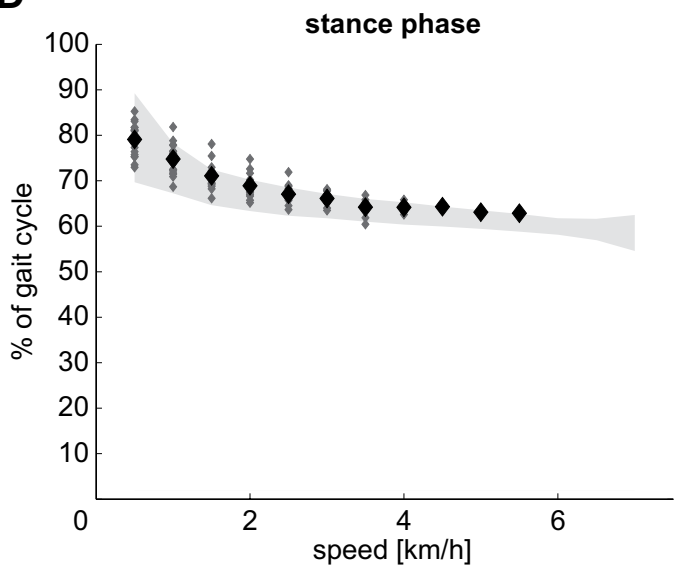

Figure 3. Speed modulation of parameters contributing to PC2 (A through D): Modulation of step length, cadence, single support, and stance phase of controls (gray area, $\pm 2 \mathrm{SD}$ interval) and patients (large dots = group mean values, small dots $=$ individual patient's data) at different walking speeds $(0.5-7.0 \mathrm{~km} / \mathrm{h}$ in controls, $0.5-5.5 \mathrm{~km} / \mathrm{h}$ in patients). The 2 groups did not differ.

Abbreviations: PC, principal component; SD, standard deviation.

could be quantified by the measure of shape difference to normal (SSD), which was strongly related to speed capacity in iSCI participants. In addition, whereas healthy participants showed a striking convergence toward a uniform pattern at preferred speed, patients could voluntarily change speed up to a certain limit but failed to improve the shape difference when increasing their walking speed. These findings indicate that speed and kinematic features respond differently to changes in supraspinal input. A similar behavior could be observed in stroke patients who showed improved gait symmetry at faster speeds but with no changes in compensatory movements. ${ }^{33}$ Despite a high responsiveness to supraspinal input at the stance to swing transition, ${ }^{34}$ speed increases were not proportionally paralleled by increased angular velocity at toe-off, suggesting that compensatory mechanisms (eg, increased hip ROM) might instead enable speed increments. The observed behaviors imply that additional factors may play a role: faster walking biomechanically induces less movement variability (ie, parallel increase of ACC and speed), ${ }^{35}$ and spasticity probably impedes the translation of increased speed to higher angular velocities.

\section{Preserved Control Mechanisms}

Despite a clear reduction in the capacity to walk beyond the preferred walking speed (ie, maximal speed), ${ }^{15}$ specific gait parameters remained unaltered in iSCI participants, suggesting less dependence on spinal cord integrity. PC2 variables represented intact interlimb coordination to produce temporally (gait-cycle phases) and spatially (step length) adequate 
A $\quad \nabla$ controls

patients

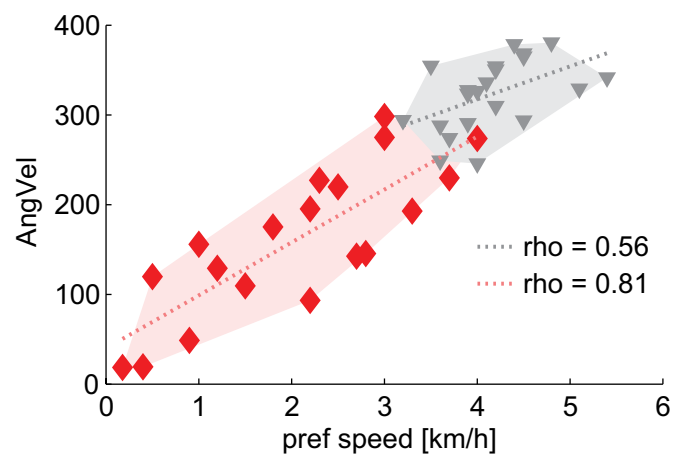

C

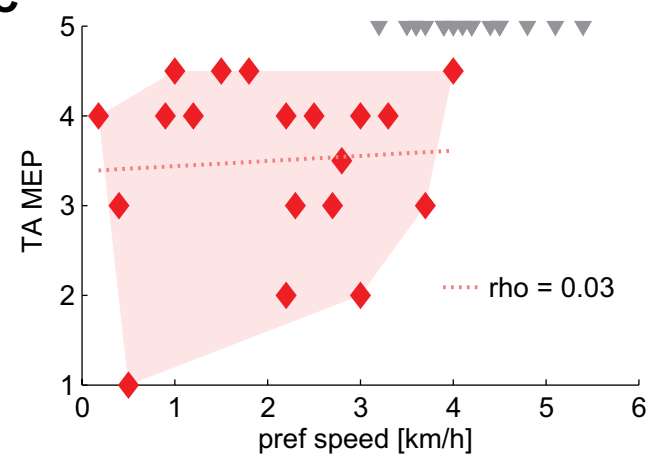

E

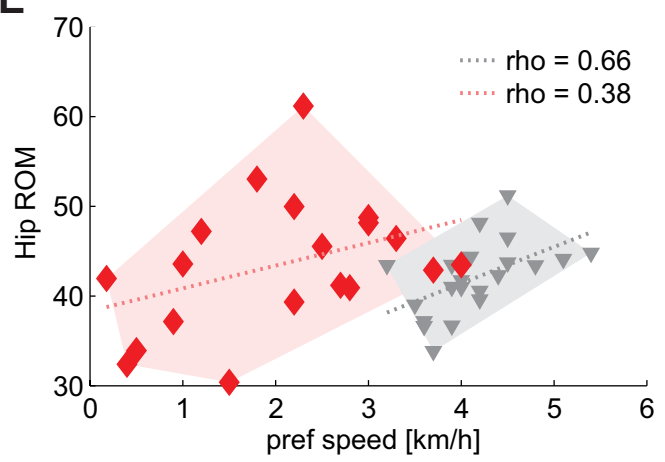

B

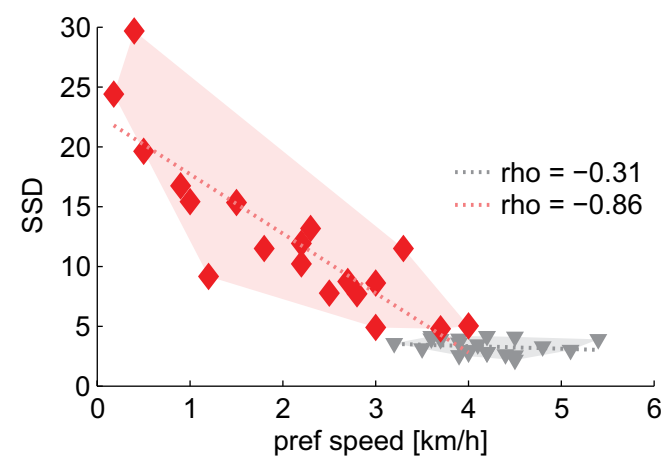

D

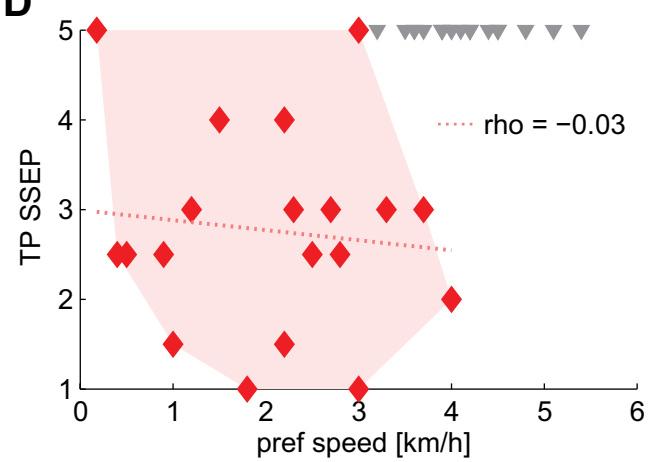

$\mathbf{F}$

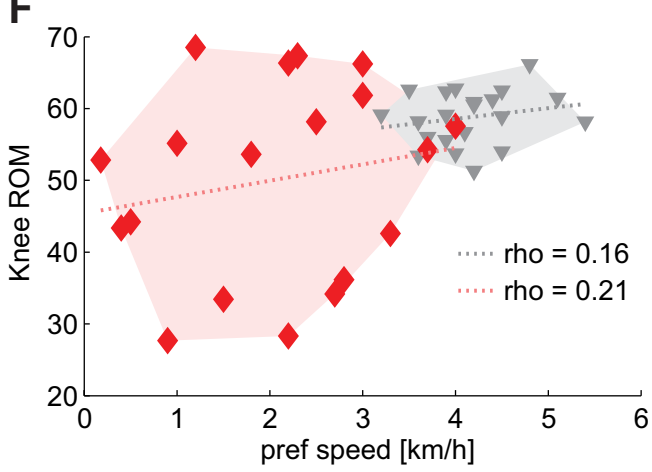

Figure 4. Relation of gait-related parameters to preferred speed: (A and B) Angular velocity at toe-off and SSD (shape difference to normal) showed linear relations to walking capacity and separated the 2 groups ( $C$ and $D)$. The degree of spinal cord integrity did not correlate with walking capacity but clearly distinguished the 2 groups. Ranges of motion showed weak to moderate relation to walking capacity and did not distinguish between groups ( $E$ and F). A linear fit was added to the graphs (dotted lines) to illustrate trends, although Spearman's correlation does not assume linearity of data.

Abbreviations: AngVel, angular velocity at toe-off; MEP, motor-evoked potential; ROM, range of motion; SSD, square root of sum of squared distances (cyclogram shape difference); SSEP, somatosensory-evoked potential; TA, tibialis anterior; TP, tibialis posterior.

Note: Color version of the figure is present with the online version of this issue at www.nnr.sagepub.com

steps, and interestingly, across the range of walking speeds that iSCI participants could attain, these parameters remained adequately modulated. This is in contrast to distinct impairments in the control of gait-cycle parameters observed in neurological disorders affecting supraspinal motor centers.
In Parkinsonian patients, step length is significantly reduced, whereas cadence can be increased to values even higher than in controls (shuffling gait). ${ }^{36}$ Stroke patients typically show an asymmetric slow gait with decreased cadence ${ }^{37}$ and limited stride length. ${ }^{38,39}$ In line with these observations, iSCI 
participants showed preserved temporal accuracy of skilled ankle movements despite diminished absolute active ROM and movement velocity. ${ }^{40}$ In contrast, stroke patients showed decreased movement accuracy both in the paretic and the unaffected leg. ${ }^{41}$ These findings indicate that muscle weakness and movement timing are unrelated, and the required control for temporal movement accuracy (within the boundaries of preserved muscle strength/speed) remains largely intact in iSCI.

\section{Domains of Locomotor Control}

The clear distinction between healthy and altered gait, which is congruent with normal and impaired spinal cord integrity assessed by spinal tract conductivity, suggests that human walking is highly dependent on unimpaired supraspinal input (cortical and subcortical). ${ }^{42}$ The nonlinear relation of measures of spinal conductivity to single gait parameters may partly be attributable to the complex interactions of supraspinal inputs that are fine-tuned by spinal networks simultaneously integrating sensory feedback from the periphery. In addition to an immediately diminished drive from supraspinal centers, spinal networks reorganize after injury and functionally change over time, ${ }^{43,44}$ which again may differently affect gait parameters. For these reasons, it remains difficult to attribute the control of specific gait parameters to particular areas of the sensorimotor system. However, one may assume that the control of distinct clusters of parameters differently depend on spinal cord integrity. Given that $\mathrm{PC} 1$ and 2 are orthogonal and, therefore, unrelated, one may conclude that PC1, comprising spinal cord integrity and speed, more strongly relies on an intact spinal cord. The parameters related to the control of reciprocal limb activation (PC2) were less dependent on spinal cord integrity and may rely more on spinal circuits (eg, central pattern generators) as shown in previous studies. ${ }^{45-49}$

\section{Implications for Interventions}

Through the advancement over the currently simplified outcome evaluation (walking speed and distance) using more comprehensive assessments combined with unbiased multivariate analysis that enables the handling of the complexity of data, patients may receive therapeutic interventions specifically tailored to their impairment. ${ }^{27}$ The identification of distinct outcome domains may allow elucidation of the role of spinal cord integrity in gait control and reveal information on underlying mechanisms involved in recovery.

\section{Limitations}

The number of participants from this inherently heterogeneous group of patients was rather small, and therefore, a generalization of results may be limited. Animal studies have revealed that voluntary changes of gait parameters, although immediately responding to supraspinal commands, are still influenced by subhierarchical (ie, spinal) effects such as facilitation or inhibition. ${ }^{7,50}$ Thus, discerning supraspinal and spinal neural control of locomotion, especially in humans, remains very challenging. A major factor influencing walking pattern is spasticity, which was not assessed in this study. In addition, the effects of medication (ie, antispasticity) and walking aids on these outcomes as well as the fact that participants were walking on a treadmill as opposed to overground require further attention.

\section{Conclusion}

Locomotor function in iSCI patients is clinically well addressed by measures of speed and distance because they represent levels of walking capacity required for daily activities but should be complemented by refined assessments of movement quality and spinal conductivity. The modulation of specific gait parameters is distinctly dependent on spinal cord integrity, and gait alterations after iSCI are specific and differ from those seen in brain disorders (eg, Parkinson's disease, stroke). The distinction and understanding of the dynamic changes of neural control after injury as well as during recovery and rehabilitation interventions may contribute to improved outcome evaluation and advanced treatments for iSCI.

\section{Acknowledgments}

We would like to thank B. Huber for assistance with patient recruitment and data acquisition and $\mathrm{W}$. Popp and $\mathrm{L}$. Tanadini for their help with statistical analyses. We would also like to thank the participants of this study.

\section{Declaration of Conflicting Interests}

The authors declared no potential conflicts of interest with respect to the research, authorship, and/or publication of this article.

\section{Funding}

The authors disclosed receipt of the following financial support for the research, authorship, and/or publication of this article: This study was supported by the European Commission's Seventh Framework Program (CP-IP 258654, NEUWalk); the Wolf Foundation, Switzerland; the Clinical Research Priority Program CRPP Neurorehab UZH; Wings for Life WFL-US-008/12, and NIH/NINDS (R01 NS067092).

\section{References}

1. Wyndaele M, Wyndaele JJ. Incidence, prevalence and epidemiology of spinal cord injury: what learns a worldwide literature survey? Spinal Cord. 2006;44:523-529.

2. Mirnezami R, Nicholson J, Darzi A. Preparing for precision medicine. N Engl J Med. 2012;366:489-491. 
3. Field-Fote EC. Combined use of body weight support, functional electric stimulation, and treadmill training to improve walking ability in individuals with chronic incomplete spinal cord injury. Arch Phys Med Rehabil. 2001;82:818-824.

4. Lucareli PR, Lima MO, Lima FP, de Almeida JG, Brech GC, D'Andrea Greve JM. Gait analysis following treadmill training with body weight support versus conventional physical therapy: a prospective randomized controlled single blind study. Spinal Cord. 2011;49:1001-1007.

5. Petersen JA, Spiess M, Curt A, Dietz V, Schubert M. Spinal cord injury: one-year evolution of motor-evoked potentials and recovery of leg motor function in 255 patients. Neurorehabil Neural Repair. 2012;26:939-948.

6. Thompson AK, Pomerantz FR, Wolpaw JR. Operant conditioning of a spinal reflex can improve locomotion after spinal cord injury in humans. $J$ Neurosci. 2013;33: 2365-2375.

7. Courtine G, Song B, Roy RR, et al. Recovery of supraspinal control of stepping via indirect propriospinal relay connections after spinal cord injury. Nat Med. 2008;14:69-74.

8. Rosenzweig ES, Courtine G, Jindrich DL, et al. Extensive spontaneous plasticity of corticospinal projections after primate spinal cord injury. Nat Neurosci. 2010;13:1505-1510.

9. van Hedel HJ, Wirz M, Curt A. Improving walking assessment in subjects with an incomplete spinal cord injury: responsiveness. Spinal Cord. 2006;44:352-356.

10. Ditunno JF Jr, Barbeau H, Dobkin BH, et al. Validity of the Walking Scale for spinal cord injury and other domains of function in a multicenter clinical trial. Neurorehabil Neural Repair. 2007;21:539-550.

11. Furlan JC, Noonan V, Singh A, Fehlings MG. Assessment of disability in patients with acute traumatic spinal cord injury: a systematic review of the literature. J Neurotrauma. 2011;28:1413-1430.

12. Field-Fote EC, Fluet GG, Schafer SD, et al. The Spinal Cord Injury Functional Ambulation Inventory (SCI-FAI). J Rehabil Med. 2001;33:177-181.

13. Lorenz DJ, Datta S, Harkema SJ. Longitudinal patterns of functional recovery in patients with incomplete spinal cord injury receiving activity-based rehabilitation. Arch Phys Med Rehabil. 2012;93:1541-1552.

14. Dobkin B, Apple D, Barbeau H, et al. Weight-supported treadmill vs over-ground training for walking after acute incomplete SCI. Neurology. 2006;66:484-493.

15. Pepin A, Norman KE, Barbeau H. Treadmill walking in incomplete spinal-cord-injured subjects: 1. Adaptation to changes in speed. Spinal Cord. 2003;41:257-270.

16. Ivanenko YP, Poppele RE, Lacquaniti F. Distributed neural networks for controlling human locomotion: lessons from normal and SCI subjects. Brain Res Bull. 2009;78:13-21.

17. Nooijen CF, Ter Hoeve N, Field-Fote EC. Gait quality is improved by locomotor training in individuals with SCI regardless of training approach. J Neuroeng Rehabil. 2009;6:36.

18. Gil-Agudo A, Perez-Nombela S, Forner-Cordero A, PerezRizo E, Crespo-Ruiz B, del Ama-Espinosa A. Gait kinematic analysis in patients with a mild form of central cord syndrome. J Neuroeng Rehabil. 2011;8:7.
19. Field-Fote EC, Tepavac D. Improved intralimb coordination in people with incomplete spinal cord injury following training with body weight support and electrical stimulation. Phys Ther. 2002;82:707-715.

20. Awai L, Curt A. Intralimb coordination as a sensitive indicator of motor-control impairment after spinal cord injury. Front Hum Neurosci. 2014;8:148.

21. Curt A, Dietz V. Traumatic cervical spinal cord injury: relation between somatosensory evoked potentials, neurological deficit, and hand function. Arch Phys Med Rehabil. 1996;77:48-53.

22. Pearson K. On lines and planes of closest fit to systems of points in space. Philos Mag. 1901;2:559-572.

23. Cattell RB. Scree test for the number of factors. Multivariate Behav Res. 1966;1:245-276.

24. Ivanenko YP, Cappellini G, Dominici N, Poppele RE, Lacquaniti F. Modular control of limb movements during human locomotion. J Neurosci. 2007;27:11149-11161.

25. Guadagnoli E, Velicer WF. Relation of sample size to the stability of component patterns. Psychol Bull. 1988;103:265-275.

26. Guadagnoli E, Velicer W. A comparison of pattern matching indices. Multivar Behav Res. 1991;26:323-343.

27. National Research Council (US) Committee on a Framework for Developing a New Taxonomy of Disease. Toward Precision Medicine: Building a Knowledge Network for Biomedical Research and a New Taxonomy of Disease. Washington, DC: National Academies Press; 2011.

28. Jacobs SR, Yeaney NK, Herbison GJ, Ditunno JF Jr. Future ambulation prognosis as predicted by somatosensory evoked potentials in motor complete and incomplete quadriplegia. Arch Phys Med Rehabil. 1995;76:635-641.

29. Zorner B, Blanckenhorn WU, Dietz V, Curt A. Clinical algorithm for improved prediction of ambulation and patient stratification after incomplete spinal cord injury. J Neurotrauma. 2010;27:241-252.

30. van Middendorp JJ, Hosman AJ, Donders AR, et al. A clinical prediction rule for ambulation outcomes after traumatic spinal cord injury: a longitudinal cohort study. Lancet. 2011;377:1004-1010.

31. Curt A, Dietz V. Ambulatory capacity in spinal cord injury: significance of somatosensory evoked potentials and ASIA protocol in predicting outcome. Arch Phys Med Rehabil. 1997;78:39-43.

32. Curt A, Keck ME, Dietz V. Functional outcome following spinal cord injury: significance of motor-evoked potentials and ASIA scores. Arch Phys Med Rehabil. 1998;79:81-86.

33. Tyrell CM, Roos MA, Rudolph KS, Reisman DS. Influence of systematic increases in treadmill walking speed on gait kinematics after stroke. Phys Ther. 2011;91:392-403.

34. Schubert M, Curt A, Jensen L, Dietz V. Corticospinal input in human gait: modulation of magnetically evoked motor responses. Exp Brain Res. 1997;115:234-246.

35. Wuehr M, Schniepp R, Pradhan C, et al. Differential effects of absent visual feedback control on gait variability during different locomotion speeds. Exp Brain Res. 2013;224: 287-294.

36. Morris M, Iansek R, Matyas T, Summers J. Abnormalities in the stride length-cadence relation in parkinsonian gait. $M o v$ Disord. 1998;13:61-69. 
37. von Schroeder HP, Coutts RD, Lyden PD, Billings E Jr, Nickel VL. Gait parameters following stroke: a practical assessment. J Rehabil Res Dev. 1995;32:25-31.

38. Nakamura R, Handa T, Watanabe S, Morohashi I. Walking cycle after stroke. Tohoku J Exp Med. 1988;154:241-244.

39. Nadeau S, Betschart M, Bethoux F. Gait analysis for poststroke rehabilitation: the relevance of biomechanical analysis and the impact of gait speed. Phys Med Rehabil Clin N Am. 2013;24:265-276.

40. Wirth B, van Hedel H, Curt A. Foot control in incomplete SCI: distinction between paresis and dexterity. Neurol Res. 2008;30:52-60.

41. van Hedel HJ, Wirth B, Curt A. Ankle motor skill is intact in spinal cord injury, unlike stroke: implications for rehabilitation. Neurology. 2010;74:1271-1278.

42. Bachmann LC, Matis A, Lindau NT, Felder P, Gullo M, Schwab ME. Deep brain stimulation of the midbrain locomotor region improves paretic hindlimb function after spinal cord injury in rats. Sci Transl Med. 2013;5:208ra146.

43. Dietz V. Behavior of spinal neurons deprived of supraspinal input. Nat Rev Neurol. 2010;6:167-174.
44. Beauparlant $\mathrm{J}$, van den Brand $\mathrm{R}$, Barraud $\mathrm{Q}$, et al. Undirected compensatory plasticity contributes to neuronal dysfunction after severe spinal cord injury. Brain. 2013;136:3347-3361.

45. Lovely RG, Gregor RJ, Roy RR, Edgerton VR. Effects of training on the recovery of full-weight-bearing stepping in the adult spinal cat. Exp Neurol. 1986;92:421-435.

46. Barbeau H, Rossignol S. Recovery of locomotion after chronic spinalization in the adult cat. Brain Res. 1987;412:84-95.

47. Dietz V, Colombo G, Jensen L. Locomotor activity in spinal man. Lancet. 1994;344:1260-1263.

48. de Leon RD, Hodgson JA, Roy RR, Edgerton VR. Locomotor capacity attributable to step training versus spontaneous recovery after spinalization in adult cats. $J$ Neurophysiol. 1998;79:1329-1340.

49. Dimitrijevic MR, Gerasimenko Y, Pinter MM. Evidence for a spinal central pattern generator in humans. Ann N Y Acad Sci. 1998;860:360-376.

50. van den Brand R, Heutschi J, Barraud Q, et al. Restoring voluntary control of locomotion after paralyzing spinal cord injury. Science. 2012;336:1182-1185. 